\title{
Detection of Indoor and Outdoor Stairs
}

\author{
Somayeh Shahrabadi, Joao M.F. Rodrigues, and J.M. Hans du Buf
}

Vision Laboratory, LARSyS, University of the Algarve, 8005-139 Faro, Portugal

\begin{abstract}
There are a few applications in which stairs must be detected, for example for aiding blind persons during navigation and for autonomous robots which must rely on vision. In this paper we present a very simple algorithm for detecting stairs, both in indoor and outdoor environments, when the camera is at a distance of approximately five meters from the stairs. The problem is hard to solve due to different factors like lighting, different constructions, materials and view angles. However, first results are very promising, although false positive and negative detections cannot yet be avoided.
\end{abstract}

Keywords: Vision, stairs, navigation, blind, autonomous robots.

\section{Introduction}

When we walk in different environments with different architectures and lighting conditions, one brief glance is enough to detect hazardous yet useful places, for example those containing stairs. This is no surprise, because our visual system is an extremely efficient and reliable pattern detector, and it has had a lot of time to learn the patterns, normally periodic sets of edges or bars. Our visual cortex even contains cells which are specialized in detecting periodic bar patterns; they are called grating cells [1].

Autonomous robots which navigate in buildings normally have special sensors for detecting steps and stairs, such that they do not bump into them like a wall or cannot fall down and be damaged. Normally these sensors suffice and are reliable, but if only one sensor fails or is accidentally blocked for some reason, the result can be disastrous. It may be a good idea that the visual functions of the robot include an occasional and rapid analysis of the environment in front to double-check the (in)existence of stairs, for example once per second.

Now imagine blind persons who cannot rely on vision. They must completely rely on the white cane, swaying it constantly in front to stay close to a wall and to detect obstacles, i.e., all possible obstacles. They too need to use stairs, and they have learned to be very cautious in environments like corridors and halls where one can expect steps and stairs. For such reasons the Blavigator project (the name combines blind and navigator) aims at developing a visual aid, basically a portable computer with a camera, in order to facilitate the life of blind persons. Being a follow-up project of SmartVision [2], during which the most basic functionality of the system was developed (path and obstacle detection; see [5]), additional functions are now being developed. One of these

J.M. Sanches, L. Micó, and J.S. Cardoso (Eds.): IbPRIA 2013, LNCS 7887, pp. 847-854, 2013.

(C) Springer-Verlag Berlin Heidelberg 2013 
concerns stairs, at a distance of a few meters which is sufficient to inform or warn the user before he or she actually reaches them with the white cane. Replacing the functionality of the normal visual system by an artificial (computer) vision system is not a simple task, because one can expect all types of complications that one can imagine: different architectures, constructions, materials, lighting and even partially occluding walls etc.

In previous work on the detection of indoor and outdoor stairs, Se and Brady 8 used a texture detection method based on Gabor filters to detect distant staircases. When close enough, staircases are then validated by looking for groups of parallel edges, where convex and concave ones are partitioned using intensity variation information. Staircase pose is estimated by a homography search approach. They employed an a priori staircase model. Lu and Manduchi [7] presented a stereo vision system to localize curbs and stairways for autonomous navigation. Their algorithm combines brightness information in the form of edges with 3D data from a commercial stereo system. In [9], Zhong et al. presented a navigation system for robot autonomous stairway climbing, as well as stairway modeling in mapping and building reconstruction, based on the combination of Gabor filters and fuzzy fusion phase grouping (FFPG).

Hernandez and Kang-Hyun [3] localized and recognized outdoor stairways by combining the longest segments of diagonal edges, the vanishing point, and Gabor filters to detect horizontal edges. Later, Hernandez et al. 4] presented a localization method for indoor stairways. Their method analyses the edges of stairs by employing planar motion tracking and directional filters. They extracted the horizontal edges by using again Gabor filters. From the specified set of horizontal edge segments, they extracted a hypothetical set by using a correlation method. Finally, they applied a discrimination method to find the ground plane on the basis of behavioral distance measurement.

Lee et al. 6] employed many small image patches in order to train (AdaBoost) a cascade classifier until true and false positive detection rates of 0.983 and $7.6 \cdot 10^{-6}$ were obtained. On real test sequences, this classifier could obtain a precision of 0.76 for recall rates up to 0.7 . However, by combining this classifier with ground plane estimation and the temporal consistency in the sequences, the precision increased to 0.84 . They mention that the total number of false positive detections was reduced from 1400 to 501. To the best of our knowledge this is the only study which presented quantitative results (see Section 4 below).

As for Se and Brady's [8] and other methods, our own method exploits the periodic structure of stairs. It is very intuitive in order to keep it as simple as possible. The main reason is that it must run in real time on small portable computers, in the future even on devices like a smart phone with a built-in camera. Our advantage is that we are not interested in the detection of stairs in general: we are only interested in stairs at small range, about $5 \mathrm{~m}$ at maximum, and in front of the user. Our results therefore provide a baseline against which later improvements can be compared. These can include the temporal consistency over consecutive image frames and ground plane estimation, perhaps also employing stereo vision [6]. 
The rest of this article is organized as follows. In the next section we describe the methods, and in Section 3 we present the results. Final conclusions and discussions are presented in the last section.

\section{Methods}

Stairs consist of series of steps with almost parallel edges, and the distance between the steps varies almost linearly. So the main goal is to extract the edges and to detect periodic edge patterns which are approximately (but not necessarily completely) horizontal. Since the system has to work in real time with a frame rate of a few frames per second, all processing must be optimized. For path and obstacle detection we have already pre-processed frames: in order to suppress noise, a frame has been filtered with a lowpass kernel, i.e., a Gaussian function $G(x, y)=\left(1 / 2 \pi \sigma^{2}\right) \cdot \exp \left\{-\left(x^{2}+y^{2}\right) / 2 \sigma^{2}\right\}$. We use a kernel size of $10 \times 10$ and $\sigma=4$. This also suppresses spurious edges before we apply the Canny edge detector with parameters $\sigma=1.0, T_{l}=0.05$ and $T_{h}=0.05$. See Fig. 1]

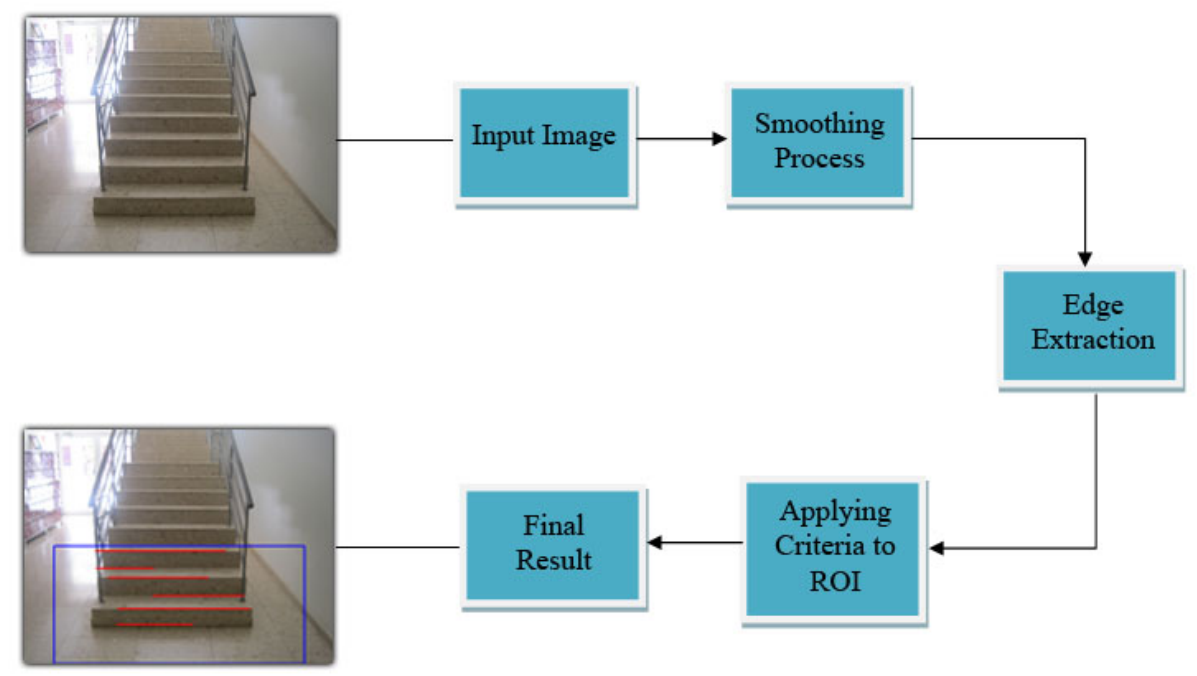

Fig. 1. Schematic diagram of the processing

Then the Hough transform is applied in order to detect edges which are approximately horizontal. This processing is done in a window or region-of-interest (ROI) which captures information in front of the camera, in the center and at the bottom part, covering the immediate vicinity until a distance of approximately 5 meters. The width and height of this ROI are $80 \%$ and $50 \%$ of the image width and height, respectively, discarding the left, right and top parts of the image. 


\subsection{Stairs Detection and Validation}

We are mainly interested in all edges (lines in the Canny edge map) that have at least one endpoint in the ROI, and horizontal and almost horizontal edges with angles between $\pm 35^{\circ}$. Horizontal and vertical surfaces of the steps may consist of differently colored or textured materials. In order to eliminate possible errors which are caused by the different materials, and to improve edge periodicity, it is necessary to apply a minimum threshold to the distances between the edges which mark parts of individual steps. After analyzing many different stairs, we apply a minimum and maximum distance of $5 \%$ and $20 \%$ of the frame height, respectively. If the distance between two edges is between the minimum and maximum distance, the edges are kept. Edges with a smaller distance are discarded and replaced by only one with the average vertical position and orientation of the discarded edges. If the distance between two edges is bigger than the maximum distance, then the edge which is closer to the bottom of the image is discarded.

Candidate stairs are detected if (1) at least 2 edges exist in the ROI, (2) each of these edges has a length of at least $20 \%$ of the ROI's width, and (3) these edges are a majority with congruent angles (they are almost parallel). When a candidate staircase has been detected, a final validation is applied. The reason is that the same edge information can be detected at crosswalks in streets, and the only difference is that crosswalks appear as almost black and white horizontal bars.

Since only edge information has been exploited in the previous steps, we now consider pixel values. First, we select a vertical region with a width of 7 pixels in the center of the ROI, and on each line the 7 pixels are averaged to reduce noise. The resulting vector is then thresholded by using the central minimum of the histogram of all pixels in the vertical region (because this region has more pixels than the average vector). Validation is positive if there are one or two black bars next to a bright one, each bar consisting of at least 10 connected vertical pixels.

\section{Results}

We emphasize that false positives and negatives cannot (yet) be avoided, because there are many quasi-periodic and "horizontal" structures, for example pavements which consist of differently-colored tiles (in- and outdoor), and (book) shelves (indoor). The data set used to test the algorithm contains images of many stairs captured at different sites, both in- and outdoor. The set contains a large variety of styles (open and solid construction, different materials), views (oblique, frontal, seen from the top and bottom floors) and distances. It also contains many images with quasi-periodic patterns like pavements and benches with shadows (see Figs 4 and 5). Figures 2 and 3 show two indoor sequences and one outdoor sequence containing stairs with different viewing angles. The ROI is indicated by the blue rectangle, and the red lines are (parts of) edges which passed the candidate test in case the stairs also passed final validation.

Of all 92 images containing stairs (positives), 76 were correctly detected and 16 were missed. Hence, the correct detection rate is $83 \%$. Of all 135 images not 

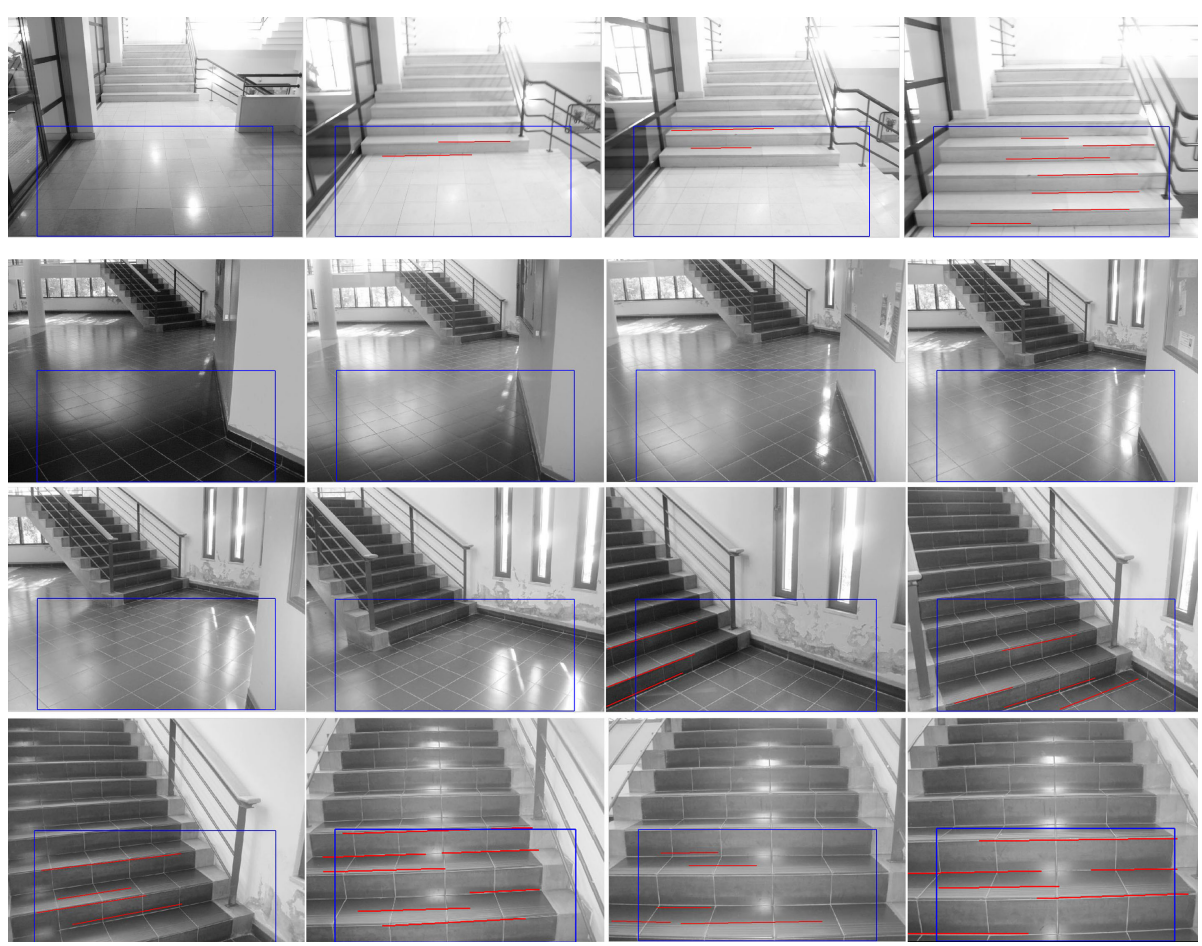

Fig. 2. Two indoor sequences containing stairs, the ROI, and detected edges after final validation

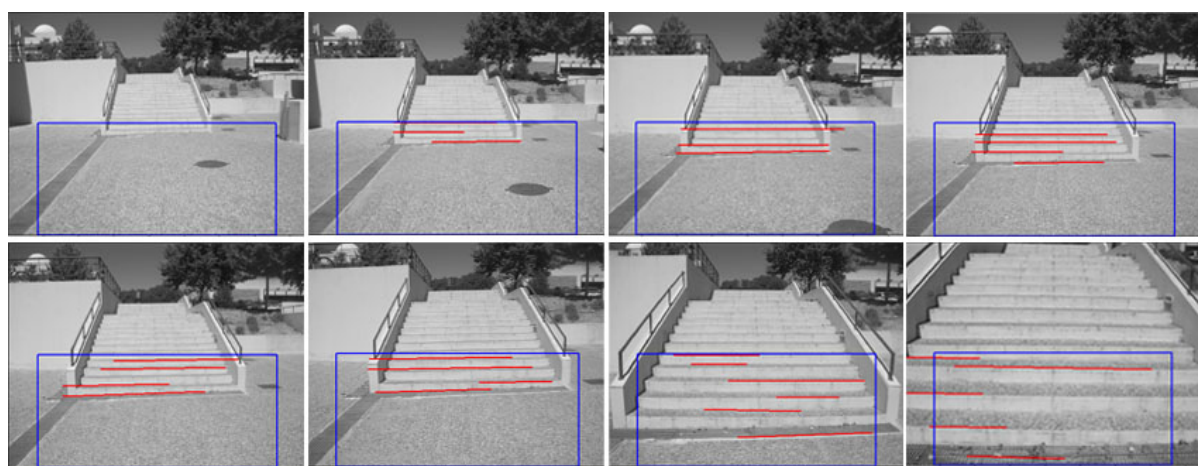

Fig. 3. An outdoor sequence containing stairs, the ROI, and detected edges after final validation 


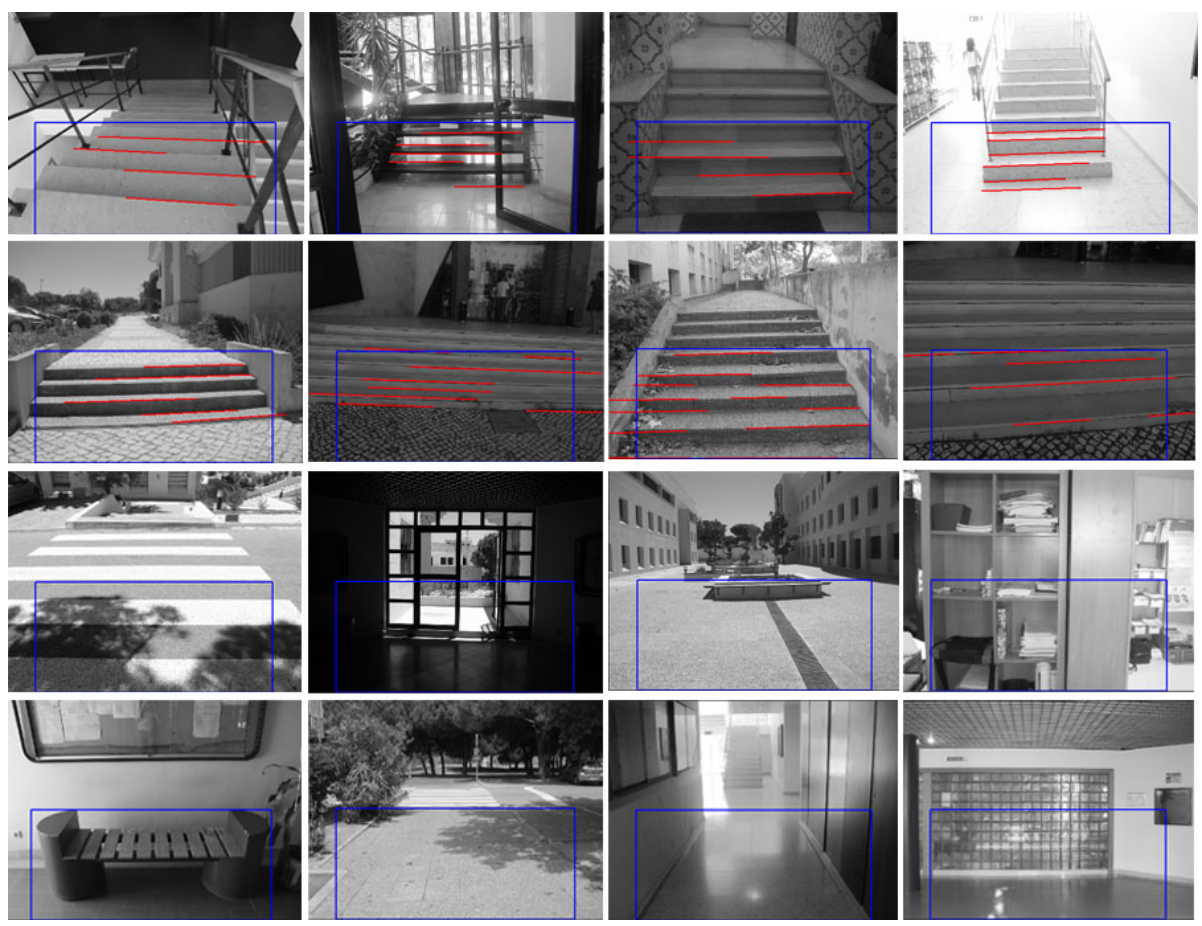

Fig. 4. Top: true positives. Bottom: true negatives

containing stairs (negatives), 110 were correctly identified as not having stairs, whereas 25 caused erroneous detection (correct rate of $81.5 \%$ ). False positives are mainly due to highly periodic non-stairs patterns and also some frames were saturated by sunshine or contained shadows. False negatives were mainly caused by insufficient lighting or low contrast due to the stairs' materials. Figure 4 shows examples of true positive and negative detections. Figure 5 shows examples of false positive and negative detections.

\section{Discussion}

As for all real-world problems, sooner or later one will encounter complications which will be very hard to solve. In this case, the problem being restricted to detecting stairs which, by definition, are always periodic structures, there is no exception to this general rule. We expected problems with many periodic patterns which are completely unrelated to stairs, like zebra street crossings etc. Indeed, outdoor detection appears more difficult than indoor detection, simply because there are many more complications like shadows, objects and tiling patterns. Also, in many cases illumination is far from perfect. Nevertheless, correct true and false recognition rates of $83 \%$ and $81.5 \%$ on a wide variety of test images (a total of 227 images) with many complications is already very good, taking into account the simplicity of the method. 

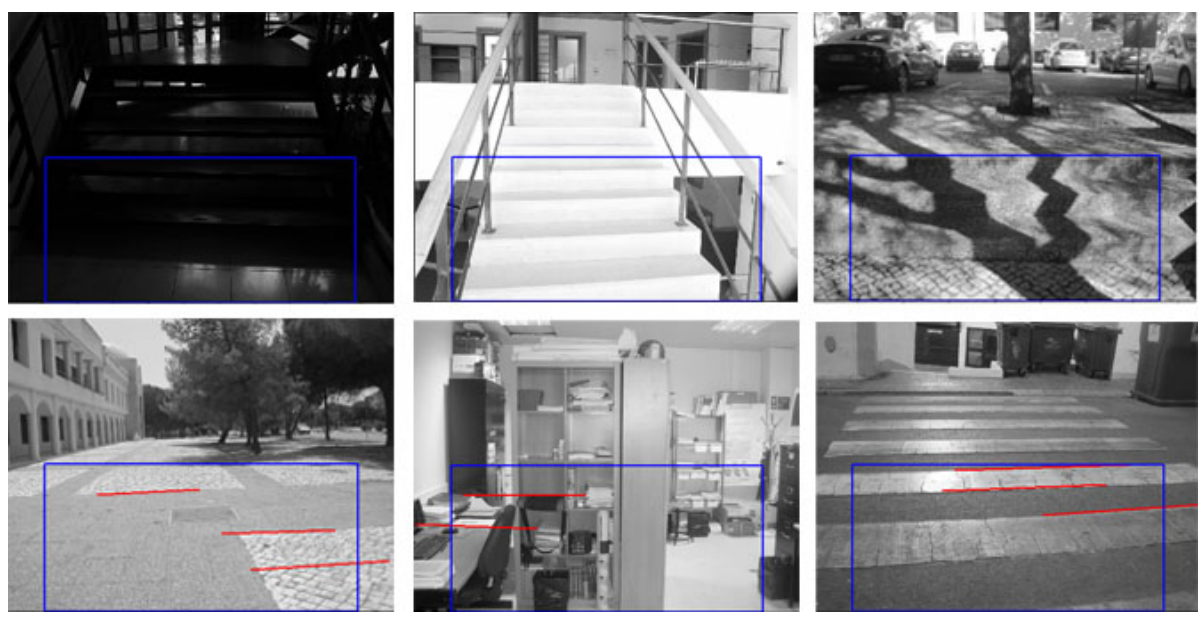

Fig. 5. Top: false negatives. Bottom: false positives

However, it is not yet good enough for being applied in practice. Until now, only periodic edge structures in "flat" images have been exploited. It seems clear that, in order to improve the method, some 3D information must be used as well. The easiest solution may be to estimate the ground plane, such that all periodic patterns on the ground, like different tiling patterns and crosswalks, can be rejected. This is subject to ongoing research. Temporal consistency over consecutive frames can be used together with estimation of the ground plane, although the latter implies stereo vision [6].

Lee et al. 6] aimed at reducing false positives, but presented results in terms of ROC curves with recall and precision statistics: a precision between 0.76 and 0.84 for recall rates under 0.7 (they also mention 501 false positives in 852 frames which contain multiple stairs). To the best of our knowledge, all other studies on the detection of stairs by using only one camera (not stereo vision or 3D sensors) did not provide quantitative results, hence a direct comparison of our results is not possible.

Acknowledgements. This work was partially supported by the Portuguese Foundation for Science and Technology (FCT) project PEstOE/EEI/LA0009/2011, EU project NeuralDynamics FP7-ICT-2009-6 PN: 270247, and FCT project Blavigator RIPD/ADA/ 109690/2009.

\section{References}

1. du Buf, J.: Improved grating and bar cell models in cortical area v1 and texture coding. Image and Vision Computing 25(6), 873-882 (2007)

2. du Buf, J., Barroso, J., Rodrigues, J., Paredes, H., Farrajota, M., Fernandes, H., José, J., Teixeira, V., Saleiro, M.: The SmartVision navigation prototype for blind users. JDCTA: Int. J. of Digital Content Technology and its Applications 5(5), $351-361$ (2011) 
3. Hernandez, D., Kang-Hyun, J.: Outdoor stairway segmentation using vertical vanishing point and directional filter. In: Proc. 2010 Int. Forum on Strategic Technology, pp. $82-86$ (2010)

4. Hernández, D.C., Kim, T., Jo, K.-H.: Stairway detection based on single camera by motion stereo. In: Mehrotra, K.G., Mohan, C.K., Oh, J.C., Varshney, P.K., Ali, M. (eds.) IEA/AIE 2011, Part I. LNCS, vol. 6703, pp. 338-347. Springer, Heidelberg (2011)

5. José, J., Rodrigues, J., du Buf, J.: Visual navigation for the blind: path and obstacle detection. In: Proc. Int. Conf. on Pattern Recognition Applications and Methods, vol. 2, pp. 515-519 (2012)

6. Lee, Y., Leung, T., Medioni, G.: Real-time staircase detection from a wearable stereo system. In: Proc. 21st Int. Conf. on Pattern Recognition, Tsukuba, Japan, November 11-15 (2012)

7. Lu, X., Manduchi, R.: Detection and localization of curbs and stairways using stereo vision. In: Proc. IEEE Int. Conf. on Robotics and Automation, pp. 4648-4654 (2005)

8. Se, S., Brady, M.: Vision-based detection of stair-cases. In: Proc. Asian Conf. on Computer Vision, pp. 535-570 (2000)

9. Zhong, C., Zhuang, Y., Wang, W.: Stairway detection using Gabor filter and FFPG. In: Proc. Int. Conf. of Soft Computing and Pattern Recognition, pp. 578-582 (2011) 